\title{
Strategies for lung isolation: to block or not to block?
}

\author{
Edmond Cohen, MD
}

Received: 14 April 2016/Accepted: 18 April 2016/Published online: 29 April 2016

(c) Canadian Anesthesiologists' Society 2016

In this issue of the Journal, Bussières et al. ${ }^{1}$ report their findings of a randomized-controlled trial evaluating the use of a bronchial blocker (BB) $v s$ a left-sided double-lumen endotracheal tube (DL-ETT) during video-assisted thoracoscopic surgery (VATS). The focus of their study was on the quality of lung collapse and the time to achieve optimal lung deflation.

The authors studied 40 patients requiring one-lung ventilation $(\mathrm{OLV})$ and randomized to receive a $\mathrm{BB}$ or a left-sided DL-ETT. The time from opening the pleura until complete lung collapse as well as the quality of lung collapse (graded from 1-3) were evaluated in real time by the surgeons who were blinded to the method used to achieve lung separation. The time to lung collapse and the quality of the collapse were also graded offline by independent observers -i.e., two thoracic surgeons and an anesthesiologist who reviewed video recordings of the VATS procedures. Finally, the operating surgeons were asked to guess which device was used for lung isolation.

The findings of the study showed that the time to complete lung collapse in patients having elective VATS was significantly faster with a BB than with a left-sided DL-ETT. The scores of the quality of lung deflation at 5, 10 , and 20 min following opening of the pleura were also better in the BB group than in the DL-ETT group, in both real-time and offline assessments. Finally, when the surgeons were asked to guess which method was being used for lung isolation, they were correct only $37 \%$ of the time. This outcome would suggest that the two devices

E. Cohen, MD ( $\square)$

Department of Anesthesiology, Icahn School of Medicine at Mount Sinai, Box 1010, One Gustave L. Levy Place, New York, NY, USA

e-mail: edmond.cohen@mountsinai.org could be used interchangeably without the surgeon noticing any differences.

As DL-ETTs are considered the "gold standard" for lung separation, they are the most widely used devices for performing this procedure. They have been used for more than 50 years, and as a result, most anesthesiologists and surgeons are familiar with them and are comfortable with their use. ${ }^{2-6}$ The decision whether to use a DL-ETT or BB depends on three factors: patient safety, the anesthesiologist's comfort with the selected device, and the surgeon's preference. Unfortunately, there are sometimes situations where these three factors cannot coincide. As with many devices, correct use of a BB involves a significant learning curve, and most anesthesiologists are unfamiliar with the device and inexperienced in its use. As a result, a thoracic surgeon who is not accustomed to having a BB used for OLV may be reluctant to accept this new technique.

The study by Bussières et al. ${ }^{1}$ addresses some of the myths surrounding the use of a BB. Indeed, there may be a bias against its use and an impression amongst surgeons (and some anesthesiologists) that a BB fails to provide optimal lung separation. ${ }^{7}$ These myths regarding the BB include the view that the quality of lung collapse is inferior to that with the DLT; that it would take more time to achieve adequate lung deflation than with the DL-ETT; and that it takes less time to insert and position a DL-ETT than to perform the same procedure with a BB. Despite these myths, the present study reinforces the points of view supported by the literature - i.e., the performance of the $\mathrm{BB}$ is at least on a par with that of the DL-ETT.

There are clearly certain clinical situations that mandate the use of a DL-ETT, particularly when the non-diseased lung must be protected from life-threatening contamination from the diseased lung. In addition, if a tumour is 
occupying the main stem bronchus or if a sleeve resection is planned, a contralateral DL-ETT should be used in almost every case.

Nevertheless, DL-ETTs are somewhat unfriendly. They can be stiff and bulky and may be difficult to insert and position properly. Over the years, anesthesiologists have found a need for simple alternative methods to achieve lung separation. There are several clinical circumstances where the use of a BB may be advantageous. Patients with a difficult airway, morbid obesity, post laryngeal surgery, or a tracheostomy present a challenge for lung separation using a DL-ETT. Perhaps the most significant advantage of the $\mathrm{BB}$ is elimination of the need to change the tracheal tube intraoperatively when indicated. Some procedures, such as an esophagectomy, descending aortic surgery, and thoracic spine surgery, are performed via a transthoracic approach and require a collapsed lung for optimal surgical exposure. These procedures can be lengthy and associated with large fluid shifts. If a patient requires postoperative ventilatory support and has a DL-ETT in place, it would be necessary to switch to a single-lumen tube, which may be challenging in the presence of upper airway edema or secretions. $^{8,9}$

In the ongoing debate concerning the performance of the BB $v s$ the DL-ETT, the literature supports the use of a BB. Narayanaswamy et al. ${ }^{10}$ evaluated the use of the Cohen blocker, Arndt blocker, Uniblocker ${ }^{\mathrm{TM}}$, and DLETT in four groups of patients. The surgeons graded the quality of lung collapse while blinded to the type of lung separation device used for OLV. The authors found no differences among the groups in the time and the quality of the lung collapse, but they did find more cuff dislocations among the BB groups.

Kosarek et al. ${ }^{11}$ reported the use of BBs to achieve lung isolation in 130 patients undergoing surgery. They used a Cohen BB in most of the patients (113/130) and a left-sided DL-ETT in 17 patients. The thoracic surgeon considered lung isolation to be adequate in all cases. The authors concluded that "the use of Cohen BB can be successful in a wide variety of thoracic operations, has minimal complications, eliminates the need for tracheal tube exchange when postoperative mechanical ventilation is required, and effectively isolates the lungs of critically ill patients." Kosarek et al.'s report is of interest, not because of the actual comparison between the two devices, but because it reveals that a BB is used almost exclusively in their institution for a wide variety of thoracic procedures, and the device provides optimal lung separation.

In their recent systematic review and comprehensive meta-analysis of randomized-controlled trials, ClaytonSmith et al. ${ }^{12}$ compared the efficacy and adverse effects of DL-ETTs $v s$ BBs in thoracic surgery. These authors concluded that DLTs could be placed more quickly and were less likely to be incorrectly positioned than BBs. Use of BBs was associated with fewer patients complaining of a postoperative sore throat, less hoarseness, and fewer airway injuries than use of DL-ETTs. Most importantly, the time taken for adequate lung collapse as well as the quality of the lung collapse showed no significant differences between the BBs and the DL-ETTs.

The work of Bussières et al. ${ }^{1}$ is extremely important in highlighting and reinforcing that the quality of lung collapse with the BB is at least as good as that with the DL-ETT, if not superior. A unique arm of the study was the offline blinded evaluation of videos to grade the quality of lung collapse at the various stages of the VATS procedures. In addition, their study confirms that adequate lung deflation with the BB was achieved as fast as with the DL-ETT, if not faster.

In contrast with other studies, Bussières et al. report a shorter time and better quality of lung collapse with the $\mathrm{BB}$, perhaps due to the two periods of apnea that were applied exclusively in the BB group. The authors speculated that lung deflation through the bronchial lumen of the DL-ETT may sometimes be compromised due to occasional obstruction of the distal portion of the bronchial lumen. Whether there is a shorter time to lung deflation with the BB than with the DL-ETT is likely of limited clinical significance. For example, a few more minutes to achieve optimal lung deflation make no difference in a VATS procedure that averages hours in duration. The take-home message from this study should be that the ability to provide safe and optimal lung separation within a reasonable time using a BB is at least on a par with that using a DL-ETT. In cases where a DL-ETT is not mandated, either device can be used to provide the surgeon with a still operating field. ${ }^{13}$

In previous reports, surgeons have graded the quality of lung collapse while blinded to the technique used for lung separation. In this study, however, the surgeons were also asked to guess which device was used for lung isolation. The fact that they were correct in only $37 \%$ of the cases shows that a surgeon's reluctance to use a BB is purely a personal bias.

Since the introduction of the Univent ${ }^{\mathrm{TM}}$ tube two decades ago, the use of $\mathrm{BBs}$ has been increasing worldwide. Four independent BBs are now approved for clinical use in North America. Nevertheless, a significant number of anesthesiologists are only vaguely familiar or comfortable with the use of a BB on a routine basis. The issue is not whether one device is superior to the other; rather, it is that anesthesia practitioners should master the use of the $\mathrm{BB}$ as an alternative option. The literature supports the use of both BBs and DL-ETTs to provide safe optimal lung separation, and the contribution by Bussières et al. clearly reinforces this concept. 


\section{Stratégies d'isolation}

\section{pulmonaire: faut-il faire un} bloc ou non?

Dans ce numéro du Journal, Bussières et coll. ${ }^{1}$ rapportent les résultats d'une étude randomisée contrôlée portant sur la qualité et le temps nécessaire à atteindre un dégonflement pulmonaire optimal. Leur étude comparait l'utilisation d'un bloqueur bronchique par rapport à une sonde endotrachéale à double lumière gauche pendant une chirurgie thoracoscopique vidéoassistée.

Les auteurs ont étudié 40 patients nécessitant une ventilation unipulmonaire et les ont randomisés à être intubés avec un bloqueur bronchique ou une sonde endotrachéale double lumière gauche. Le temps entre l'ouverture pleurale et le dégonflement complet du poumon, ainsi que la qualité du dégonflement pulmonaire (notée de 1 à 3), ont été évalués en temps réel par les chirurgiens, lesquels ignoraient la méthode utilisée pour isoler le poumon. Le temps jusqu'au dégonflement du poumon et la qualité du dégonflement ont également été notés hors ligne par des observateurs indépendants (c.-à-d. deux chirurgiens thoraciques et un anesthésiologiste) qui ont passé en revue les enregistrements vidéo des chirurgies thoracoscopiques vidéoassistées. Enfin, on a demandé au chirurgien ayant pratiqué l'intervention de deviner quel dispositif avait été utilisé pour la séparation pulmonaire.

Les résultats de l'étude ont montré que, chez les patients subissant une chirurgie thoracique vidéoassistée non urgente, le temps jusqu'au dégonflement pulmonaire était significativement plus court avec un bloqueur bronchique qu'avec une sonde endotrachéale double lumière gauche. Les scores de qualité du dégonflement pulmonaire à cinq, dix et vingt minutes après l'ouverture de la plèvre étaient également meilleurs dans le groupe bloqueur bronchique, tant lors des évaluations en temps réel que dans les évaluations hors ligne. Enfin, lorsqu'on a demandé aux chirurgiens de deviner quelle méthode avait été utilisée pour la séparation pulmonaire, leurs réponses étaient correctes dans seulement $37 \%$ des cas. On peut donc penser que les deux dispositifs seraient interchangeables et que le chirurgien ne remarquerait pas de différence.

Les sondes endotrachéales double lumière sont considérées «l'étalon or » de la séparation pulmonaire, c'est pourquoi elles sont les dispositifs les plus utilisés pour isoler un poumon. Elles sont utilisées depuis plus de 50 ans; la plupart des anesthésiologistes et des chirurgiens les connaissent donc bien et savent bien les utiliser. ${ }^{2-6} \mathrm{La}$ décision d'avoir recours à une sonde endotrachéale double lumière ou un bloqueur bronchique se fonde sur trois facteurs: la sécurité des patients, l'aise de l'anesthésiologiste avec le dispositif choisi, et la préférence $\mathrm{du}$ chirurgien. Malheureusement, dans certaines situations, ces trois facteurs sont irréconciliables. Comme c'est le cas pour bon nombre de dispositifs, la courbe d'apprentissage du bloqueur bronchique est considérable; ainsi, la plupart des anesthésiologistes ne sont pas familiers avec son utilisation et n'ont pas beaucoup d'expérience en la matière. Par conséquent, un chirurgien thoracique qui n'a pas l'habitude d'utiliser un bloqueur bronchique pour réaliser une ventilation unipulmonaire pourrait être réticent à accepter cette nouvelle technique.

L'étude de Bussières et coll. ${ }^{1}$ traite également de certains des mythes entourant l'utilisation des bloqueurs bronchiques. En effet, il pourrait y avoir un biais contre l'utilisation d'un bloqueur bronchique ou une croyance, parmi les chirurgiens (et certains anesthésiologistes), qu'un bloqueur bronchique ne peut procurer une séparation pulmonaire optimale. ${ }^{7}$ Parmi ces mythes, citons la croyance que la qualité du dégonflement pulmonaire n'est pas aussi bonne qu'avec une sonde double lumière; qu'il faudrait plus de temps pour obtenir un dégonflement pulmonaire adéquat avec un bloqueur bronchique; et qu'il faut moins de temps pour insérer et positionner une sonde endotrachéale double lumière. Et pourtant, malgré ces mythes, l'étude présentée ici vient renforcer ce que la littérature nous disait déjà : la performance d'un bloqueur bronchique est au moins aussi bonne que celle d'une sonde endotrachéale double lumière.

Il est clair que certaines situations requièrent l'utilisation d'une sonde endotrachéale double lumière, particulièrement lorsque le poumon non lésé doit être protégé d'une contamination potentiellement fatale du poumon malade. En outre, si une tumeur occupe la bronche souche, ou si une résection en manchon est prévue, il convient, dans la majorité des cas, d'utiliser une sonde endotrachéale double lumière controlatérale.

Toutefois, les sondes endotrachéales double lumière sont peu conviviales, peuvent être rigides, volumineuses et difficiles à insérer et positionner correctement. Au fil des ans, les anesthésiologistes se sont rendus compte qu'ils avaient besoin d'alternatives simples pour isoler un poumon. Un bloqueur bronchique pourrait être utile dans plusieurs circonstances cliniques. La séparation pulmonaire peut être un défi chez les patients présentant des voies aériennes difficiles ou une obésité morbide, ceux subissant une chirurgie post-laryngée ou encore ceux ayant une trachéostomie, si elle est réalisée avec une sonde endotrachéale double lumière. Avec un bloqueur bronchique, il n'est plus nécessaire de changer la sonde trachéale pendant l'opération lorsque cela est indiqué : c'est probablement là son avantage le plus important. Certaines interventions, comme par exemple les 
œsophagectomies, les chirurgies de l'aorte descendante ou celles de la colonne thoracique, sont réalisées par approche transthoracique; elles nécessitent donc un poumon dégonflé, de façon à obtenir une exposition chirurgicale optimale. Ces interventions peuvent être longues et sont associées à d'importants transferts liquidiens. Si le patient a besoin d'un soutien ventilatoire postopératoire et qu'on a utilisé une sonde endotrachéale double lumière, il faut la remplacer par une sonde simple lumière, ce qui pourrait être ardu en cas d'œdème facial ou de sécrétions. ${ }^{8,9}$

Dans le débat incessant concernant la performance d'un bloqueur bronchique par rapport à une sonde endotrachéale double lumière, la littérature penche en faveur du bloqueur bronchique. Narayanaswamy et coll. ${ }^{10}$ ont évalué l'utilisation de bloqueurs bronchiques de type Cohen, Arndt et Uniblocker ainsi que d'une sonde double lumière dans quatre groupes de patients. L'évaluation notée de la qualité de dégonflement du poumon a été réalisée par les chirurgiens, lesquels ignoraient quel dispositif de séparation pulmonaire avait été utilisé. Les auteurs n'ont pas trouvé de différence d'un groupe à l'autre en matière de temps et de qualité du dégonflement pulmonaire; toutefois, ils ont observé que le nombre de déplacements du ballonnet était plus élevé dans les groupes des bloqueurs bronchiques.

Kosarek et coll. ${ }^{11}$ ont évalué l'utilisation de bloqueurs bronchiques pour réaliser l'isolation pulmonaire de 130 patients subissant une chirurgie. Un bloqueur de type Cohen a été utilisé chez la plupart des patients (113/130), mais chez 17 patients, on a utilisé une sonde endotrachéale double lumière gauche. Le chirurgien thoracique a jugé l'isolation pulmonaire adéquate dans tous les cas. Les auteurs concluent que «l'utilisation de bloqueurs bronchiques peut réussir dans diverses opérations thoraciques, qu'ils entraînent un minimum de complications, éliminent le besoin d'échanger la sonde trachéale lorsqu'on a besoin d'une ventilation mécanique postopératoire, et qu'ils isolent efficacement les poumons des patients gravement malades. » Le compte rendu de Kosarek et coll. est intéressant, non pas à cause de la comparaison de deux dispositifs, mais parce qu'il démontre que, dans l'institution où exercent ces auteurs, le bloqueur bronchique est le dispositif de choix pour toutes sortes d'interventions thoraciques et qu'il permet une séparation pulmonaire optimale.

Clayton-Smith et coll. ${ }^{12}$ ont récemment publié une revue méthodique et une méta-analyse exhaustive des études randomisées contrôlées comparant l'efficacité et les effets secondaires néfastes des sondes endotrachéales double lumière et des bloqueurs bronchiques en chirurgie thoracique. Ces auteurs concluent que les sondes double lumière sont plus rapides à placer et qu'il est moins probable qu'elles soient mal positionnées que les bloqueurs bronchiques. L'utilisation de bloqueurs bronchiques était associée à un plus petit nombre de patients se plaignant de maux de gorge, d'enrouement et de lésions aux voies aériennes en période postopératoire que les sondes endotrachéales double lumière. Mais, plus important encore, aucune différence significative n'a été observée entre les bloqueurs bronchiques et les sondes double lumière en ce qui concernait le temps nécessaire à obtenir un dégonflement pulmonaire adéquat et la qualité du dégonflement pulmonaire.

Les travaux de Bussières et coll. ${ }^{1}$ sont extrêmement importants, car ils soulignent et renforcent que la qualité du dégonflement pulmonaire obtenue avec un bloqueur bronchique est au moins aussi bonne qu'avec une sonde double lumière, voire supérieure. La composante unique de cette étude réside dans son évaluation en aveugle et hors ligne des vidéos pour noter la qualité du dégonflement pulmonaire aux différentes étapes des interventions de chirurgie thoracoscopique vidéoassistée. En outre, cette étude confirme qu'un dégonflement pulmonaire adéquat peut être obtenu avec un bloqueur bronchique aussi rapidement, voire plus rapidement encore, qu'avec une sonde endotrachéale double lumière.

L'étude de Bussières et coll. est la seule à rapporter un délai plus court et une meilleure qualité de dégonflement pulmonaire avec un bloqueur bronchique. Ces résultats sont peut-être dus aux deux périodes d'apnée appliquées exclusivement aux patients du groupe intubé avec un bloqueur bronchique. Selon les auteurs, il est possible que le dégonflement du poumon à travers la lumière bronchique de la sonde endotrachéale double lumière pourrait parfois être compromis en raison d'une obstruction occasionnelle de la portion distale de la lumière bronchique. L'impact clinique de la réduction du temps nécessaire jusqu'au dégonflement pulmonaire avec un bloqueur bronchique est probablement limité. En effet, il est fort possible que quelques minutes supplémentaires pour obtenir un dégonflement pulmonaire optimal ne feront aucune différence dans une intervention de chirurgie thoracoscopique vidéoassistée qui dure en général plusieurs heures. Le message à retenir de cette étude devrait être qu'un bloqueur bronchique peut procurer une séparation pulmonaire sécuritaire et optimale dans un temps raisonnable tout aussi efficacement qu'une sonde endotrachéale double lumière. Dans les cas où une sonde endotrachéale double lumière n'est pas absolument nécessaire, l'un ou l'autre dispositif peut donc être utilisé pour procurer au chirurgien un champ opératoire immobile. $^{13}$

L'évaluation de la qualité du dégonflement pulmonaire par des chirurgiens ne connaissant pas la technique utilisée pour isoler le poumon avait déjà été rapportée. Dans l'étude présentée ici toutefois, les chirurgiens ont 
également dû deviner quel dispositif avait été utilisé. Le fait qu'ils n'aient répondu correctement que dans $37 \%$ des cas montre que la réticence d'un chirurgien à utiliser un bloqueur bronchique réside uniquement dans son biais personnel.

Depuis l'introduction de la sonde Univent ${ }^{\circledR}$ il y a vingt ans, l'utilisation des bloqueurs bronchiques a augmenté partout dans le monde. Quatre bloqueurs bronchiques indépendants sont maintenant approuvés pour utilisation clinique en Amérique du Nord. Toutefois, de nombreux anesthésiologistes ne les connaissent pas bien et ne se sentent pas à l'aise de les utiliser. La question n'est pas de savoir si un dispositif est meilleur que l'autre, mais plutôt que les anesthésiologistes devraient maîtriser l'utilisation d'un bloqueur bronchique comme alternative. La littérature soutient l'utilisation tant des bloqueurs bronchiques que des sondes endotrachéales double lumière pour procurer une séparation pulmonaire sécuritaire et optimale, et la contribution de Bussières et coll. renforce clairement cette notion.

Conflicts of interest None declared.

Editorial responsibility This submission was handled by Dr. Hilary P. Grocott, Editor-in-Chief, Canadian Journal of Anesthesia.

\section{Conflit d'intérêt Aucun.}

Responsabilité éditoriale Cet article a été traité par Dr Hilary P. Grocott, rédacteur en chef, Journal canadien d'anesthésie.

\section{References}

1. Bussières JS, Somma J, Carrasco del Castillo JL, et al. Bronchial blocker versus left double-lumen endotracheal tube in videoassisted thoracoscopic surgery: a randomized-controlled trial examining timing and quality of lung deflation. Can $\mathbf{J}$ Anesth 2016; 63. DOI: $10.1007 / \mathrm{s} 12630-016-0657-3$.

2. Cohen E. Management of one-lung ventilation. Anesthesiol Clin North America 2001; 19: 475-95.

3. Eisenkraft JB, Neustein S, Cohen E. Anesthesia for Thoracic Surgery. In: Barash PG, Cullen BF, Stoelting RK, Cahalan M, Stock MC, Ortega R, editors. Handbook of Clinical Anesthesia 7th ed. Philadelphia, PA: JB Lippincott Company; 2013. p. 103075.

4. Cohen E. Recommendations for airway control and difficult management in thoracic anesthesia and lung separation procedures. Are we ready for the challenge? Minerva Anestesiol 2009; 75: 3-5.

5. Brodsky JB, Lemmens HJ. Left double-lumen tubes: clinical experience with 1,170 patients. J Cardiothorac Vasc Anesth 2003; 17: 289-98.

6. Campos JH. Progress in lung separation. Thorac Surg Clin 2005; 15: 71-83.

7. Brodsky JB. Con: a bronchial blocker is not a substitute for a double-lumen endobronchial tube. J Cardiothorac Vasc Anesth 2015; 29: 237-9.

8. Dumans-Nizard V, Liu N, Laloe PA, Fischler M. A comparison of the deflecting-tip bronchial blocker with a wire-guided blocker or left-sided double-lumen tube. J Cardiothorac Vasc Anesth 2009; 23: 501-5.

9. Cohen E. Back to blockers: the continued search for the ideal endobronchial blocker. Anesthesiology 2013; 118: 490-3.

10. Narayanaswamy $M$, McRae $K$, Slinger $P$, et al. Choosing a lung isolation device for thoracic surgery: a randomized trial of three bronchial blockers versus double-lumen tubes. Anesth Analg 2009; 108: 1097-101.

11. Kosarek L, Busch E, Abbas A, Falterman J, Nossaman B. Effective use of bronchial blockers in lung isolation surgery: an analysis of 130 cases. Ochsner J 2013; 13: 389-93.

12. Clayton-Smith A, Bennett $K$, Alston RP, et al. A comparison of the efficacy and adverse effects of double-lumen endobronchial tubes and bronchial blockers in thoracic surgery: a systematic review and meta-analysis of randomized controlled trials. J Cardiothorac Vasc Anesth 2015; 29: 955-66.

13. Yoo JY, Kim DH, Choi H, Kim K, Chae YJ, Park SY. Disconnection technique with a bronchial blocker for improving lung deflation: a comparison with a double-lumen tube and bronchial blocker without disconnection. J Cardiothorac Vasc Anesth 2014; 28: 904-7. 